\title{
Frame-Theoretic Analysis of Oversampled Filter Banks
}

\author{
Helmut Bölcskei, Member, IEEE, Franz Hlawatsch, Member, IEEE, and Hans G. Feichtinger
}

\begin{abstract}
We provide a frame-theoretic analysis of oversampled finite impulse response (FIR) and infinite impulse response (IIR) uniform filter banks (FB's). Our analysis is based on a new relationship between the FB's polyphase matrices and the frame operator corresponding to an FB. For a given oversampled analysis FB, we present a parameterization of all synthesis FB's providing perfect reconstruction. We find necessary and sufficient conditions for an oversampled FB to provide a frame expansion. A new frame-theoretic procedure for the design of paraunitary FB's from given nonparaunitary FB's is formulated. We show that the frame bounds of an FB can be obtained by an eigenanalysis of the polyphase matrices. The relevance of the frame bounds as a characterization of important numerical properties of an FB is assessed by means of a stochastic sensitivity analysis. We consider special cases in which the calculation of the frame bounds and synthesis filters is simplified. Finally, simulation results are presented.
\end{abstract}

Index Terms - Filter banks, frames, oversampling, polyphase representation.

\section{INTRODUCTION}

$\mathbf{U}$ NIFORM filter banks (FB's), ${ }^{1}$ i.e., filter banks with the same decimation factor in each channel [1]-[7], correspond to a class of discrete-time signal expansions. The relation between discrete-time signal expansions and maximally decimated (or critically sampled) FB's has been studied in [1], [2], [8], and [9]. It has also been recognized that oversampled FB's [2], [4], [7], [10] correspond to redundant signal expansions [2], [11]-[21]. Oversampled FB's have recently received increased attention due to their improved design freedom [17], [21]-[23], and noise immunity [21]-[23]. These advantages of oversampled FB's come at the expense of increased computational cost. Thus, oversampled FB's allowing an efficient implementation, such as oversampled DFT FB's [4], [7], [11], [16], [21], [24], [25] and oversampled cosine modulated FB's [21], [24], [26], are of particular interest.

The theory of frames [25], [27]-[33] is an appropriate mathematical framework for redundant signal expansions. Due to the correspondence between redundant signal expansions

Manuscript received March 1, 1996; revised April 22, 1998. The associate editor coordinating the review of this paper and approving it for publication was Dr. Jelena Kovacević.

H. Bölcskei and F. Hlawatsch are with the Institut für Nachrichtentechnik und Hochfrequenztechnik, Vienna University of Technology, Vienna, Austria (e-mail: hboelcsk@aurora.nt.tuwien.ac.at; fhlawats@email.tuwien.ac.at).

H. G. Feichtinger is with the Department of Mathematics, NUHAG, University of Vienna, Vienna, Austria (e-mail: fei@tyche.mat.univie.ac.at).

Publisher Item Identifier S 1053-587X(98)08696-6.

${ }^{1}$ For the sake of brevity, we shall use the term filter bank (FB) instead of uniform filter bank. and oversampled FB's, the theory of frames is similarly appropriate for oversampled FB's. The use of frame theory for the study of oversampled FIR FB's was first proposed by Cvetković and Vetterli [11], [12], [20] and has also been discussed in [13]-[19].

In this paper, we present a frame-theoretic approach to oversampled FB's [14]-[17] that is based on an extension of the Zibulski-Zeevi method for the analysis of continuous-time Weyl-Heisenberg frames [34]-[36]. Our approach extends previous work reported in [11]-[13] and [18] and leads to several further original results that include

- a parameterization of all synthesis FB's providing perfect reconstruction (PR) for a given oversampled analysis FB;

- methods for estimating the frame bounds of an FB, constructing paraunitary FB's from nonparaunitary FB's and calculating approximations to PR synthesis FB's;

- a stochastic sensitivity analysis for oversampled FB's involving the frame bounds.

In addition, we show that certain results formulated in [11]-[13] and [18] for the FIR case also hold in the IIR case. Our approach is based on the fact (to be shown in the paper) that the FB's polyphase matrices provide matrix representations of the frame operator corresponding to an FB. This fundamental result allows an efficient frame-theoretic analysis of oversampled FIR and IIR FB's.

We shall now outline the paper's organization and main results. Section II briefly reviews oversampled FB's and their connection to frames. Section III shows that the polyphase matrices provide matrix representations of a FB's frame, analysis, and synthesis operators; these matrix representations will furnish a basis for most of our subsequent results. In Section IV, a parameterization of all synthesis FB's providing PR for a given oversampled analysis FB is presented, and a condition for completeness is given. Section V formulates necessary and sufficient conditions for an oversampled FIR or IIR FB to correspond to a frame. A stochastic sensitivity analysis highlighting the importance of the frame bounds is provided, it is shown how the frame bounds can be estimated from the polyphase matrices, and the approximative construction of the minimum norm PR synthesis FB is discussed. In Section VI, we show that oversampled paraunitary FB's correspond to tight frames, and we propose a new method for constructing paraunitary FB's from given nonparaunitary FB's. Section VII considers important special cases where the calculation of the minimum norm synthesis FB and the frame bounds is simplified. Finally, simulation results are presented in Section VIII. 


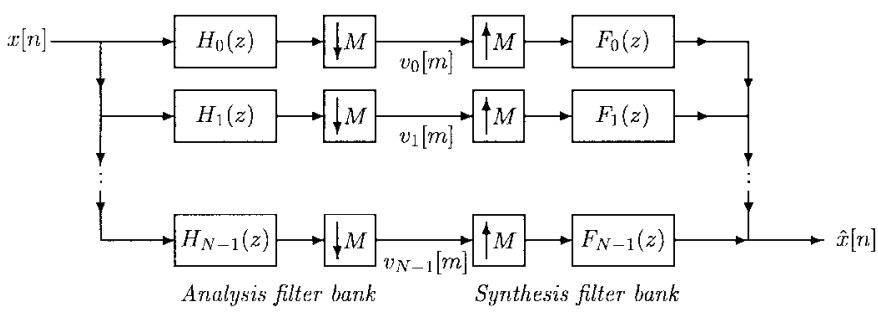

Fig. 1. $\quad N$-channel uniform filter bank.

\section{OVERSAMPLED FILTER BANKS AND FRAMES}

In this section, we briefly review oversampled FB's and their relation to frames in $l^{2}(\mathbb{Z})$. We discuss the type of frames corresponding to FB's, and we show that if the analysis frame is of that type, then so is the dual synthesis frame.

\section{A. Oversampled FB's}

We consider an $N$-channel FB (see Fig. 1) with subsampling by the integer factor $M$ in each channel. The transfer functions of the analysis and synthesis filters are $H_{k}(z)$ and $F_{k}(z)$ ( $k=0,1, \cdots, N-1$ ), with corresponding impulse responses $h_{k}[n]$ and $f_{k}[n]$, respectively. The subband signals are given by

$$
\begin{aligned}
v_{k}[m] & =(\boldsymbol{E} x)[m, k] \\
& =\sum_{n=-\infty}^{\infty} x[n] h_{k}[m M-n] \\
& =\left\langle x, h_{k, m}\right\rangle, \quad k=0,1, \cdots, N-1
\end{aligned}
$$

with $h_{k, m}[n]=h_{k}^{*}[m M-n](k=0,1, \cdots, N-1,-\infty<$ $m<\infty)$, and the reconstructed signal is

$$
\hat{x}[n]=(\boldsymbol{R v})[n]=\sum_{k=0}^{N-1} \sum_{m=-\infty}^{\infty} v_{k}[m] f_{k, m}[n]
$$

with $f_{k, m}[n]=f_{k}[n-m M]$ and $\mathbf{v}[m]=$

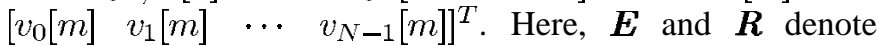
the FB analysis and synthesis operator, respectively.

In the critically sampled (or maximally decimated) case $N=$ $M$, the subband signals $v_{k}[m]$ contain exactly as many samples (per unit of time) as the input signal $x[n]$. In the oversampled case $N>M$, however, the subband signals are redundant in that they contain more samples (per unit of time) than the input signal $x[n]$. Oversampled FB's offer more design freedom and improved numerical properties as compared with critically sampled FB's, and they have noise-reducing properties [17], [21]-[23]. The design freedom is increased since for a given oversampled analysis FB, there exists a whole class of synthesis FB's providing PR (see Section IVA). The noise-reducing properties of redundant representations [21]-[23], [28], [37] allow a coarser quantization of the subband signals at the cost of increased sample rate [21]-[23] (see Section V-C).

Our frame-theoretic analysis of oversampled FB's will be based on the well-known polyphase representation [1], [2], [5], [38] of FB's or, equivalently, the discrete Zak transform [39]-[42]. The polyphase decomposition of the analysis filters
$H_{k}(z)$ reads

$$
H_{k}(z)=\sum_{n=0}^{M-1} z^{n} E_{k, n}\left(z^{M}\right)
$$

with

$$
E_{k, n}(z)=\sum_{m=-\infty}^{\infty} h_{k}[m M-n] z^{-m} .
$$

The $N \times M$ analysis polyphase matrix $\mathbf{E}(z)$ is defined as $[\mathbf{E}(z)]_{k, n}=E_{k, n}(z)$. The synthesis filters $F_{k}(z)$ can be similarly decomposed as

$$
F_{k}(z)=\sum_{n=0}^{M-1} z^{-n} R_{k, n}\left(z^{M}\right)
$$

with

$$
R_{k, n}(z)=\sum_{m=-\infty}^{\infty} f_{k}[m M+n] z^{-m} .
$$

The $M \times N$ synthesis polyphase matrix $\mathbf{R}(z)$ is defined as $[\mathbf{R}(z)]_{n, k}=R_{k, n}(z)$.

\section{B. Uniform FB Frames}

If the FB satisfies PR with zero delay, ${ }^{2}$ i.e., $\hat{x}[n]=x[n]$, then (2) yields

$$
x[n]=\sum_{k=0}^{N-1} \sum_{m=-\infty}^{\infty}\left\langle x, h_{k, m}\right\rangle f_{k, m}[n] .
$$

This shows that a PR FB corresponds to an expansion of the input signal $x[n]$ into the function set $\left\{f_{k, m}[n]\right\}$ (k= $0,1, \cdots, N-1,-\infty<m<\infty)$ [1], [2], [43]. In general, the set $\left\{f_{k, m}[n]\right\}$ is not orthogonal; therefore, the expansion coefficients, i.e., the subband signals $v_{k}[m]=\left\langle x, h_{k, m}\right\rangle$, are obtained by projecting the signal $x[n]$ onto a "dual" set of functions $\left\{h_{k, m}[n]\right\}$. Critically sampled FB's provide orthogonal or biorthogonal signal expansions [43], whereas oversampled FB's correspond to redundant (overcomplete) expansions [2], [11]-[21].

The theory of frames [25], [27]-[33] is a powerful vehicle for the study of redundant signal expansions. The set $\left\{h_{k, m}[n]\right\}$ is said to be a frame for $l^{2}(\mathbb{Z})$ if ${ }^{3}$

$$
\begin{array}{r}
A\|x\|^{2} \leq \sum_{k=0}^{N-1} \sum_{m=-\infty}^{\infty}\left|\left\langle x, h_{k, m}\right\rangle\right|^{2} \leq B\|x\|^{2} \\
\forall x[n] \in l^{2}(\mathbb{Z})
\end{array}
$$

with the frame bounds $A>0$ and $B<\infty$. The frame bounds determine important numerical properties of the FB as discussed in Sections V-C and V-D. If the analysis set $\left\{h_{k, m}[n]\right\}$ is a frame for $l^{2}(\mathbb{Z})$, then PR can always be achieved, and a particular synthesis set providing PR is given by (see [28] and [30])

$$
f_{k, m}[n]=\left(\boldsymbol{S}^{-1} h_{k, m}\right)[n] .
$$

\footnotetext{
${ }^{2}$ We note that our theory can easily be extended to PR with nonzero delay. ${ }^{3}$ Here, $l^{2}(\mathbb{Z})$ denotes the space of square-summable functions $x[n]$, i.e., $\sum_{n=-\infty}^{\infty}|x[n]|^{2}<\infty$.
} 
Here, $S^{-1}$ is the inverse of the frame operator of $\left\{h_{k, m}[n]\right\}$ defined as

$$
(\boldsymbol{S} x)[n]=\sum_{k=0}^{N-1} \sum_{m=-\infty}^{\infty}\left\langle x, h_{k, m}\right\rangle h_{k, m}[n] .
$$

The frame operator is a positive definite, linear operator that maps $l^{2}(\mathbb{Z})$ onto $l^{2}(\mathbb{Z})$. It can be written as $\boldsymbol{S}=\boldsymbol{E}^{* *} \boldsymbol{E}$, where $\boldsymbol{E}$ is the analysis operator in (1), and $\boldsymbol{E}^{*}$ is its adjoint [44]. The frame bounds $A$ and $B$ are the infimum and supremum, respectively, of the eigenvalues of $S$ [28], [30].

If the analysis set $\left\{h_{k, m}[n]\right\}$ is a frame, then the synthesis set $\left\{f_{k, m}[n]\right\}$ defined by (4) is also a frame (the "dual" frame) with frame operator $S^{-1}$ and frame bounds $A^{\prime}=1 / B$, $B^{\prime}=1 / A$. A frame is called snug if $B / A=B^{\prime} / A^{\prime} \approx 1$ and tight if $B / A=B^{\prime} / A^{\prime}=1$. For a tight frame, we have $S^{-1}=(1 / A) \boldsymbol{I}$, where $\boldsymbol{I}$ is the identity operator on $l^{2}(\mathbb{Z})$; hence, there is simply $f_{k, m}[n]=(1 / A) h_{k, m}[n]$.

The analysis and synthesis frames corresponding to (uniform) FB's have a specific "shift invariant" structure since $h_{k, m}[n]=h_{k}^{*}[-(n-m M)]$ and $f_{k, m}[n]=f_{k}[n-m M]$, i.e., they are generated by uniformly time shifting the FB impulse responses $h_{k}^{*}[-n]$ and $f_{k}[n]$. A frame with such a shift-invariant structure will be called a uniform filter bank frame (UFBF). Such frames have also been considered in [12], [13], [19], and (in a continuous-time setting) in [45].

FB's whose analysis functions $h_{k, m}[n]$ satisfy the frame condition (3) and whose synthesis functions $f_{k, m}[n]$ are chosen as the dual frame of $h_{k, m}[n]$ provide UFBF expansions. If $\left\{h_{k, m}[n]\right\}$ is a UFBF, then the dual frame $\left\{f_{k, m}[n]\right\}$ as defined by (4) is again a UFBF, i.e., it is generated by uniformly time shifting a dual set of functions given by $f_{k}[n]=$ $\left(S^{-1} \widetilde{h}_{k}\right)[n]$ with $\tilde{h}_{k}[n]=h_{k}^{*}[-n]$. This can be seen as follows. Introducing the unitary time-shift operator $\boldsymbol{T}_{m}$ as $\left(\boldsymbol{T}_{m} x\right)[n]=$ $x[n-m M]$, we can write $h_{k, m}[n]=\left(\boldsymbol{T}_{m} \tilde{h}_{k}\right)[n]$. Using $\boldsymbol{T}_{m} \boldsymbol{T}_{m^{\prime}}=\boldsymbol{T}_{m+m^{\prime}}$ and $\boldsymbol{T}_{m}^{*}=\boldsymbol{T}_{-m}$ (with $\boldsymbol{T}_{m}^{*}$ denoting the adjoint of $\boldsymbol{T}_{m}$ ), it is easily shown that both the frame operator $S$ and its inverse $S^{-1}$ commute with the time-shift operator $T_{m}$, i.e., $T_{m} S=S T_{m}$ and $\boldsymbol{T}_{m} \boldsymbol{S}^{-1}=\boldsymbol{S}^{-1} \boldsymbol{T}_{m}$. We then obtain $f_{k, m}[n]=\left(\boldsymbol{S}^{-1} h_{k, m}\right)[n]=\left(\boldsymbol{S}^{-1} \boldsymbol{T}_{m} \tilde{h}_{k}\right)[n]=$ $\left(T_{m} S^{-1} \tilde{h}_{k}\right)[n]=\left(T_{m} f_{k}\right)[n]=f_{k}[n-m M]$ with $f_{k}[n]=$ $\left(S^{-1} \tilde{h}_{k}\right)[n]$.

Even though our frame-theoretic approach is valid both for oversampling and critical sampling, in this paper, we restrict our attention to oversampled FB's and the corresponding UFBF's. We just note that the frames corresponding to critically sampled FB's are exact, i.e., orthogonal or biorthogonal function sets [8], [43].

\section{MATRIX REPRESENTATIONS}

Important problems in frame theory include the inversion of the frame operator $S$ and the calculation of frame bounds $A, B$. In this section, we will show that the FB's frame operator $\boldsymbol{S}$, analysis operator $\boldsymbol{E}$, and synthesis operator $\boldsymbol{R}$ can be expressed in terms of the FB's polyphase matrices. Thus, the inversion of the frame operator and the calculation of the frame bounds can be reduced to operations involving the polyphase matrices.

\section{A. Matrix Representation of the Frame Operator}

The following result extends the Zibulski-Zeevi representation of continuous-time Weyl-Heisenberg frame operators [34]-[36].

Lemma 3.1: Let $y[n]=(\boldsymbol{S} x)[n]$, where $\boldsymbol{S}$ is the frame operator corresponding to a UFBF. Then, the polyphase components $Y_{n}(z)=\sum_{m=-\infty}^{\infty} y[m M+n] z^{-m}$ of $Y(z)$ and the polyphase components $X_{n^{\prime}}(z)=\sum_{m=-\infty}^{\infty} x\left[m M+n^{\prime}\right] z^{-m}$ of $X(z)$ are related as

$$
Y_{n}(z)=\sum_{n^{\prime}=0}^{M-1} S_{n, n^{\prime}}(z) X_{n^{\prime}}(z)
$$

with

$$
S_{n, n^{\prime}}(z)=\sum_{k=0}^{N-1} \tilde{E}_{k, n}(z) E_{k, n^{\prime}}(z)
$$

or equivalently, using the polyphase vectors $\mathrm{x}(z)=\left[X_{0}(z)\right.$ $\left.X_{1}(z) \quad \cdots \quad X_{M-1}(z)\right]^{T}$ and $\mathbf{y}(z)=\left[\begin{array}{lll}Y_{0}(z) & Y_{1}(z) & \cdots\end{array}\right.$ $\left.Y_{M-1}(z)\right]^{T}$

$$
\mathbf{y}(z)=\mathbf{S}(z) \mathbf{x}(z) \quad \text { with } \mathbf{S}(z)=\tilde{\mathbf{E}}(z) \mathbf{E}(z)
$$

where $\tilde{\mathbf{E}}(z)=\mathbf{E}^{H}\left(1 / z^{*}\right)$ denotes $^{4}$ the paraconjugate of $\mathbf{E}(z)$ [1].

Proof: Evaluating the polyphase components $Y_{n}(z)=$ $\sum_{m=-\infty}^{\infty} y[m M+n] z^{-m}$ of the signal $y[n]=(\boldsymbol{S} x)[n]=$ $\sum_{k=0}^{N=1} \sum_{m=-\infty}^{\infty}\left\langle x, h_{k, m}\right\rangle h_{k, m}[n],(5)$ is obtained after simple manipulations.

Thus, the frame operator $S$ can be expressed in the polyphase domain by the $M \times M U F B F$ matrix $\mathbf{S}(z)=$ $\tilde{\mathbf{E}}(z) \mathbf{E}(z)$ defined in terms of the analysis polyphase matrix $\mathbf{E}(z)$.

Specializing to the unit circle $\left(z=e^{j 2 \pi \theta}\right)$, we next show that the polyphase matrix $\mathbf{E}\left(e^{j 2 \pi \theta}\right)$ can be used to establish a matrix representation [44] of the frame operator $S$. Most of our subsequent discussion of FB's will be based on this matrix representation.

Theorem 3.1: Let $S$ be the frame operator corresponding to a UFBF. Then, the $M \times M$ matrix

$$
\mathbf{S}\left(e^{j 2 \pi \theta}\right)=\mathbf{E}^{H}\left(e^{j 2 \pi \theta}\right) \mathbf{E}\left(e^{j 2 \pi \theta}\right)
$$

is positive definite for all $\theta$; furthermore, it is the matrix representation of the frame operator $S$ with respect to the basis $\left\{e_{n, \theta}\left[n^{\prime}\right]\right\}$ of $l^{2}(\mathbb{Z})$ given by $e_{n, \theta}\left[n^{\prime}\right]=\sum_{m=-\infty}^{\infty} \delta\left[n^{\prime}-n-\right.$ $m M] e^{j 2 \pi(\theta / M)\left(n^{\prime}-n\right)}(n=0,1, \cdots, M-1,0 \leq \theta<1)$.

Proof: Using $X_{n}\left(e^{j 2 \pi \theta}\right)=\left\langle x, e_{n, \theta}\right\rangle$, it follows after straightforward manipulations that

$$
\left\langle S x, e_{n, \theta}\right\rangle=\sum_{n^{\prime}=0}^{M-1} S_{n, n^{\prime}}\left(e^{j 2 \pi \theta}\right)\left\langle x, e_{n^{\prime}, \theta}\right\rangle .
$$

This shows that $\mathbf{S}\left(e^{j 2 \pi \theta}\right)=\mathbf{E}^{H}\left(e^{j 2 \pi \theta}\right) \mathbf{E}\left(e^{j 2 \pi \theta}\right)$ is the matrix representation of $S$ with respect to the basis $e_{n, \theta}\left[n^{\prime}\right]$. The

\footnotetext{
${ }^{4}$ The superscript ${ }^{H}$ stands for conjugate transposition.

${ }^{5}$ This basis induces the polyphase representation on the unit circle: $\left\langle x, e_{n, \theta}\right\rangle=X_{n}\left(e^{j 2 \pi \theta}\right)=\sum_{m=-\infty}^{\infty} x[m M+n] e^{-j 2 \pi \theta m}$. Equivalently, $\left\langle x, e_{n, \theta}\right\rangle$ is the Zak transform of $x[n]$ [39]-[42].
} 
positive definiteness of $\mathbf{S}\left(e^{j 2 \pi \theta}\right)$ then follows from the positive definiteness of $\boldsymbol{S}$.

An important consequence of Theorem 3.1 is the identity of the eigenvalues of the frame operator with the eigenvalues of its matrix representation: the UFBF matrix.

Corollary 3.1: Let $\lambda_{n}(\theta)$ with $n=0,1, \cdots, M-1$ denote the eigenvalues of the UFBF matrix $\mathbf{S}\left(e^{j 2 \pi \theta}\right)=$ $\mathbf{E}^{H}\left(e^{j 2 \pi \theta}\right) \mathbf{E}\left(e^{j 2 \pi \theta}\right)$, which are defined by the eigenequation

$$
\begin{aligned}
\mathbf{S}\left(e^{j 2 \pi \theta}\right) \mathbf{u}_{n}(\theta) & =\lambda_{n}(\theta) \mathbf{u}_{n}(\theta) \\
n & =0,1, \cdots, M-1,0 \leq \theta<1 .
\end{aligned}
$$

Any eigenvalue $\lambda_{n}(\theta)$ is simultaneously an eigenvalue of the frame operator $S$. Conversely, any eigenvalue of $S$ is simultaneously an eigenvalue of $\mathbf{S}\left(e^{j 2 \pi \theta}\right)$.

Proof: Using (6), it can easily be shown that $S=$ $\mathcal{Z}^{-1} \mathbf{S}\left(e^{j 2 \pi \theta}\right) \mathcal{Z}$, where $\mathcal{Z}: x \rightarrow X_{n}\left(e^{j 2 \pi \theta}\right)=\left\langle x, e_{n, \theta}\right\rangle=$ $\sum_{m=-\infty}^{\infty} x[m M+n] e^{-j 2 \pi \theta m}$ denotes the polyphase transform (Zak transform) operator, i.e., the operator mapping a signal to the polyphase domain with $z=e^{j 2 \pi \theta}$. Since $\mathcal{Z}$ is a unitary transformation [39], it follows that $\boldsymbol{S}$ and $\mathbf{S}\left(e^{j 2 \pi \theta}\right)$ are unitarily equivalent. Therefore, $S$ and $\mathbf{S}\left(e^{j 2 \pi \theta}\right)$ have the same eigenvalues [44].

It follows that the eigenanalysis of the frame operator $S$ (a matrix of infinite size) is equivalent to that of the UFBF matrix $\mathbf{S}\left(e^{j 2 \pi \theta}\right)$ [an $M \times M$ matrix indexed by a real-valued parameter $\theta \in[0,1)]$. Since $\mathbf{S}\left(e^{j 2 \pi \theta}\right)$ is a positive definite matrix, its eigenvalues are positive. These results will be used for the estimation of frame bounds in Section V-A.

\section{B. Matrix Representation of the Analysis and Synthesis Operators}

According to (1), the analysis operator $\boldsymbol{E}$ maps the input signal $x[n]$ into the subband signals $v_{k}[m]$. Transforming (1) into the $z$-transform domain yields

$$
\mathbf{v}(z)=\mathbf{E}(z) \mathbf{x}(z)
$$

where $\mathbf{v}(z)=\sum_{m=-\infty}^{\infty} \mathbf{v}[m] z^{-m}$ and $\mathbf{x}(z)=$ $\left[\begin{array}{lllll}X_{0}(z) & X_{1}(z) & \cdots & X_{M-1}(z)\end{array}\right]^{T}$ with $X_{n}(z)=$ $\sum_{m=-\infty}^{\infty} x[m M+n] z^{-m}$. Thus, the analysis polyphase matrix $\mathbf{E}(z)$ provides a polyphase domain representation of the analysis operator $\boldsymbol{E}$. Comparing $S=\boldsymbol{E}^{*} \boldsymbol{E}$ with $\mathbf{S}(z)=\tilde{\mathbf{E}}(z) \mathbf{E}(z)$, it is furthermore clear that the adjoint analysis operator $\boldsymbol{E}^{*}$ is represented by the paraconjugate $\tilde{\mathbf{E}}(z)$.

In a similar manner, transforming (2) into the polyphase domain yields

$$
\hat{\mathbf{x}}(z)=\mathbf{R}(z) \mathbf{v}(z)
$$

where $\hat{\mathbf{x}}(z)=\left[\hat{X}_{0}(z) \hat{X}_{1}(z) \cdots \hat{X}_{M-1}(z)\right]^{T}$ with $\hat{X}_{n}(z)=$ $\sum_{m=-\infty}^{\infty} \hat{x}[m M+n] z^{-m}$. This shows that the synthesis operator $R$ is represented in the polyphase domain by the synthesis polyphase matrix $\mathbf{R}(z)$.

\section{Perfect Reconstruction And Completeness}

We will now derive a parameterization of all synthesis FB's providing PR for a given oversampled analysis FB. Furthermore, we will formulate a necessary and sufficient condition for completeness of the analysis set $\left\{h_{k, m}[n]\right\}$, which is a prerequisite for PR. In this section, we do not assume that the FB corresponds to a frame, even though close relations to frame theory will become evident.

\section{A. Parameterization of All Perfect \\ Reconstruction Synthesis FB's}

In the oversampled case $(N>M)$, the synthesis FB providing PR for a given analysis FB is not uniquely determined. This nonuniqueness entails a desirable freedom of design that does not exist in the case of critical sampling. The following theorem provides a parameterization of all PR synthesis FB's corresponding to a given analysis FB.

Theorem 4.1: Let $\mathbf{E}(z)$ denote the analysis polyphase matrix in an oversampled $\mathrm{FB}$, and assume that $\mathbf{E}(z)$ has full rank, i.e., $\operatorname{rank}\{\mathbf{E}(z)\}=M$, almost everywhere (a.e.). Then, all synthesis polyphase matrices $\mathbf{R}(z)$ providing PR can be written as

$$
\mathbf{R}(z)=\mathbf{R}^{(0)}(z)+\mathbf{U}(z)\left[\mathbf{I}_{N}-\mathbf{E}(z) \mathbf{R}^{(0)}(z)\right]
$$

where $\mathbf{R}^{(0)}(z)$ is any particular PR synthesis polyphase matrix, i.e., any left inverse of $\mathbf{E}(z)$, and $\mathbf{U}(z)$ is an $M \times N$ matrix with arbitrary elements $[\mathbf{U}(z)]_{n, k}$ satisfying $\left|\left[\mathbf{U}\left(e^{j 2 \pi \theta}\right)\right]_{n, k}\right|<\infty$. A special choice for $\mathbf{R}^{(0)}(z)$ is the para-pseudoinverse of $\mathbf{E}(z)$, which is defined as ${ }^{6}$

$$
\hat{\mathbf{R}}(z)=[\tilde{\mathbf{E}}(z) \mathbf{E}(z)]^{-1} \tilde{\mathbf{E}}(z) .
$$

Proof: It is well known that an oversampled or critically sampled FB provides PR (with zero delay) if and only if

$$
\mathbf{R}(z) \mathbf{E}(z)=\mathbf{I}_{M} .
$$

In the oversampled case $(N>M)$, the matrices $\mathbf{E}(z)$ and $\mathbf{R}(z)$ are rectangular ( $N \times M$ and $M \times N$, respectively), and thus, the solution $\mathbf{R}(z)$ of (9) [for given $\mathbf{E}(z)$ ] is not uniquely determined; in fact, any left inverse of $\mathbf{E}(z)$ is a valid solution. Specializing results from linear algebra [47, p. 46], it follows that any left inverse of $\mathbf{E}(z)$ can be written as in (7), where $\mathbf{R}^{(0)}(z)$ is any particular solution of (9). It is straightforward to verify that the para-pseudoinverse in (8) satisfies (9) and is, thus, a valid solution.

Expression (7) is a parameterization of $\mathbf{R}(z)$ in terms of the $M N$ parameters $[\mathrm{U}(z)]_{n, k}$ that can be chosen arbitrarily. Note that the family of PR synthesis polyphase matrices $\mathbf{R}(z)$ corresponds to a family of PR synthesis filters $f_{k}[n]$. The importance of the parameterization (7) lies in the fact that once we know some left inverse $\mathbf{R}^{(0)}(z)$ [such as the para-pseudoinverse $\left.\hat{\mathbf{R}}(z)=[\tilde{\mathbf{E}}(z) \mathbf{E}(z)]^{-1} \tilde{\mathbf{E}}(z)\right]$, the optimum design of the PR synthesis FB for a given oversampled analysis FB can be performed using an unconstrained optimization. That is, the PR property need no longer be explicitly incorporated in the optimization as a side constraint. This leads to considerable simplifications in optimum FB design.

The particular PR synthesis FB corresponding to the parapseudoinverse $\hat{\mathbf{R}}(z)=[\tilde{\mathbf{E}}(z) \mathbf{E}(z)]^{-1} \tilde{\mathbf{E}}(z)$ can be given an

${ }^{6} \mathrm{We}$ note that on the unit circle, the para-pseudoinverse in (8) becomes the conventional pseudoinverse [46] $\hat{\mathbf{R}}\left(e^{j 2 \pi \theta}\right)=$ $\left[\mathbf{E}^{H}\left(e^{j 2 \pi \theta}\right) \mathbf{E}\left(e^{j 2 \pi \theta}\right)\right]^{-1} \mathbf{E}^{H}\left(e^{j 2 \pi \theta}\right)$. 
interesting frame-theoretic interpretation, which has previously been described for the important class of oversampled FIR FB's in [12]. For given analysis filter impulse responses $h_{k}[n]$, consider the particular synthesis filter impulse responses $f_{k}[n]$ provided by frame theory via (4), i.e., $f_{k}[n]=\left(S^{-1} \tilde{h}_{k}\right)[n]$ with $\tilde{h}_{k}[n]=h_{k}^{*}[-n]$, or in other words, $\left\{f_{k, m}[n]\right\}$ is the $\mathrm{UFBF}$ that is dual to $\left\{h_{k, m}[n]\right\}$. From $\tilde{h}_{k}[n]=\left(S f_{k}\right)[n]$, it follows with (6) that $\tilde{\mathbf{E}}(z)=\tilde{\mathbf{E}}(z) \mathbf{E}(z) \mathbf{R}(z)$ [simply set $x[n]=f_{k}[n]$ and $y[n]=\tilde{h}_{k}[n]$ in (6) for $\left.k=0,1, \cdots, N-1\right]$. This implies $\mathbf{R}(z)=[\tilde{\mathbf{E}}(z) \mathbf{E}(z)]^{-1} \tilde{\mathbf{E}}(z)=\hat{\mathbf{R}}(z)$. Thus, the para-pseudoinverse of $\mathbf{E}(z)$ corresponds to the particular PR synthesis FB provided by frame theory. This frametheoretic solution has minimum norm in the sense that it minimizes $\sum_{k=0}^{N-1}\left\|f_{k}\right\|^{2}$ among the class of all PR synthesis FB's [28], [30]. Using $\mathbf{S}(z)=\tilde{\mathbf{E}}(z) \mathbf{E}(z)$ and $\hat{\mathbf{R}}(z)=$ $[\tilde{\mathbf{E}}(z) \mathbf{E}(z)]^{-1} \tilde{\mathbf{E}}(z)$, it furthermore follows that the matrix representation of the inverse frame operator $S^{-1}$ is given by $\mathbf{S}^{-1}(z)=\hat{\mathbf{R}}(z) \hat{\mathbf{R}}(z)$. We note that the relation between pseudoinverses and frames has been established in a different context in [48] and [49].

The parameterization (7) can be reformulated in the time domain as

$$
f_{k}[n]=f_{k}^{(0)}[n]+u_{k}[n]-\sum_{l=0}^{N-1} \sum_{m=-\infty}^{\infty}\left\langle f_{k}^{(0)}, h_{l, m}\right\rangle u_{l, m}[n]
$$

where the $f_{k}^{(0)}[n]$ are the PR synthesis filter impulse responses corresponding to the polyphase matrix $\mathbf{R}^{(0)}(z), u_{k}[n]$ is the impulse response of the filter with polyphase components $[\mathbf{U}(z)]_{n, k}$, i.e., $U_{k}(z)=\sum_{n=0}^{M-1} z^{-n}\left[\mathbf{U}\left(z^{M}\right)\right]_{n, k}$, and $u_{k, m}[n]=u_{k}[n-m M]$. In the $z$-transform domain, (7) can be reformulated as

$$
\begin{aligned}
F_{k}(z)= & F_{k}^{(0)}(z)+U_{k}(z)-\frac{1}{M} \sum_{i=0}^{M-1} F_{k}^{(0)}\left(z W_{M}^{i}\right) \\
& \cdot\left[\sum_{l=0}^{N-1} H_{l}\left(z W_{M}^{i}\right) U_{l}(z)\right]
\end{aligned}
$$

where $W_{M}=e^{-j 2 \pi / M}$. Thus, all PR synthesis filters are parameterized in terms of the $N$ filters $u_{k}[n] \leftrightarrow U_{k}(z)$ that can be chosen arbitrarily. In the following, we will mainly use the minimum norm synthesis $F B \hat{\mathbf{R}}(z)$ or, equivalently, $\left\{\hat{f}_{k}[n]\right\}$, which is obtained by setting $\mathbf{R}^{(0)}(z)=\hat{\mathbf{R}}(z)$ and $\mathbf{U}(z)=0$ in $(7)$.

\section{B. Completeness Condition}

The next theorem states a condition for the completeness of the analysis set $\left\{h_{k, m}[n]\right\}$. The completeness of $\left\{h_{k, m}[n]\right\}$ is a necessary condition for $\mathrm{PR}$, as well as a necessary condition for the frame property (cf. Section V-B).

Theorem 4.2: The set $\left\{h_{k, m}[n]\right\}$ with $h_{k, m}[n]=$ $h_{k}^{*}[m M-n]$ is complete in $l^{2}(\mathbb{Z})$ if and only if the analysis polyphase matrix $\mathbf{E}\left(e^{j 2 \pi \theta}\right)$ has full rank, i.e., $\operatorname{rank}\left\{\mathbf{E}\left(e^{j 2 \pi \theta}\right)\right\}=M$, a.e. on $\theta \in[0,1)$.
Proof: Assuming completeness of $\left\{h_{k, m}[n]\right\}$, it follows that $\langle S x, x\rangle=\sum_{k=0}^{N-1} \sum_{m=-\infty}^{\infty}\left|\left\langle x, h_{k, m}\right\rangle\right|^{2}>0$ for all $x[n] \neq 0$ and, hence, the eigenvalues of $S$ [simultaneously the eigenvalues $\lambda_{n}(\theta)$ of $\mathbf{S}\left(e^{j 2 \pi \theta}\right)$; see Corollary 3.1] satisfy $\lambda_{n}(\theta)>0$ a.e. for $0 \leq \theta<1$ and $n=0,1, \cdots, M-1$. This shows that $\mathbf{S}\left(e^{j 2 \pi \theta}\right)$ has full rank a.e., i.e., $\operatorname{rank}\left\{\mathbf{S}\left(e^{j 2 \pi \theta}\right)\right\}=$ $M$ a.e. Using $\operatorname{rank}\left\{\mathbf{S}\left(e^{j 2 \pi \theta}\right)\right\}=\operatorname{rank}\left\{\mathbf{E}\left(e^{j 2 \pi \theta}\right)\right\}$ [47], it follows that $\operatorname{rank}\left\{\mathbf{E}\left(e^{j 2 \pi \theta}\right)\right\}=M$ a.e. on $\theta \in[0,1)$. The converse statement is shown by reversing this line of reasoning.

With $\operatorname{rank}\left\{\mathbf{S}\left(e^{j 2 \pi \theta}\right)\right\}=\operatorname{rank}\left\{\mathbf{E}\left(e^{j 2 \pi \theta}\right)\right\}$ [47], it immediately follows that an equivalent condition for completeness is $\operatorname{rank}\left\{\mathbf{S}\left(e^{j 2 \pi \theta}\right)\right\}=M$ a.e. on $\theta \in[0,1)$.

It is intuitively obvious that FB's cannot satisfy the PR property in the undersampled case $N<M$ since there are fewer subband samples (per unit of time) than input samples. Indeed, for $N<M$, the set $\left\{h_{k, m}[n]\right\}$ is incomplete in $l^{2}(\mathbb{Z})$. This is so because for $N<M$, the rank of the $N \times M$ matrix $\mathbf{E}\left(e^{j 2 \pi \theta}\right)$ is maximally $N$. Hence, $\operatorname{rank}\left\{\mathbf{S}\left(e^{j 2 \pi \theta}\right)\right\}=$ $\operatorname{rank}\left\{\mathbf{E}\left(e^{j 2 \pi \theta}\right)\right\}<M$, and using Theorem 4.2, it follows that $\left\{h_{k, m}[n]\right\}$ is incomplete in $l^{2}(\mathbb{Z})$.

\section{FrAme-ThEORETIC PROPERTIES}

As mentioned in Section II-B, FB's providing UFBF expansions are always PR FB's. Besides the fact that the frame property implies the PR property, it is also desirable since it guarantees a certain degree of numerical stability (see the stochastic sensitivity analysis in Section V-C). This section discusses frame-theoretic aspects of FB's in terms of the matrix representations developed in Section III. We will present a method for estimating the frame bounds and conditions guaranteeing that an FIR or IIR FB corresponds to a UFBF expansion. Furthermore, a stochastic sensitivity analysis involving the frame bounds will be provided, and the approximative construction of the PR synthesis FB with minimum norm will be discussed.

\section{A. Frame Bounds}

Since the frame bounds describe important numerical properties of a FB, their calculation is of interest. The next corollary states that the frame bounds follow from the eigenvalues of the UFBF matrix.

Corollary 5.1: The (tightest possible) frame bounds $A$ and $B$ of a FB providing a UFBF expansion are given by the essential infimum and supremum, respectively, of the eigenvalues $\lambda_{n}(\theta)$ of the UFBF matrix $\mathbf{S}\left(e^{j 2 \pi \theta}\right)=\mathbf{E}^{H}\left(e^{j 2 \pi \theta}\right) \mathbf{E}\left(e^{j 2 \pi \theta}\right)$ :

$$
\begin{aligned}
& A=\underset{\theta \in[0,1), n=0,1, \cdots, M-1}{\operatorname{essinf}} \lambda_{n}(\theta) \\
& B=\underset{\theta \in[0,1), n=0,1, \cdots, M-1}{\operatorname{ess} \sup _{n}} \lambda_{n}(\theta) .
\end{aligned}
$$

Proof: It is well known [28], [30], [33] that the (tightest possible) frame bounds $A$ and $B$ are the essential infimum and the essential supremum, respectively, of the eigenvalues of the frame operator $\boldsymbol{S}$. Hence, Corollary 5.1 follows using Corollary 3.1.

Similarly, we have $A^{\prime}=\operatorname{ess~}_{\inf _{\theta \in[0,1)}, n=0,1, \cdots, M-1} \lambda_{n}^{\prime}(\theta)$ and $B^{\prime}=$ ess $\sup _{\theta \in[0,1), n=0,1, \cdots, M-1} \lambda_{n}^{\prime}(\theta)$, where the $\lambda_{n}^{\prime}(\theta)$ 
are the eigenvalues of the inverse UFBF matrix $\mathbf{S}^{-1}\left(e^{j 2 \pi \theta}\right)=$ $\hat{\mathbf{R}}\left(e^{j 2 \pi \theta}\right) \hat{\mathbf{R}}^{H}\left(e^{j 2 \pi \theta}\right)$. Note that in practice, the frame bounds have to be estimated by sampling the matrix $\mathbf{S}\left(e^{j 2 \pi \theta}\right)$ on the unit circle and performing an eigenanalysis of $\mathbf{S}\left(e^{j 2 \pi l / L}\right)$ for $l=0,1, \cdots, L-1$. In Section VII, we will discuss situations where the frame bounds can be calculated without performing an explicit eigenanalysis.

An interesting consequence of Corollary 5.1 is the following corollary, which has been formulated for the FIR case in [13] and is extended below to the IIR case.

Corollary 5.2: Let $\left\{h_{k, m}[n]\right\}$ be a UFBF for $l^{2}(\mathbb{Z})$ with frame bounds $A$ and $B$. Then

$$
A \leq \frac{1}{M} \sum_{k=0}^{N-1}\left\|h_{k}\right\|^{2} \leq B .
$$

In particular, in the case of a tight UFBF (where $A=B$ ), we have

$$
\frac{1}{M} \sum_{k=0}^{N-1}\left\|h_{k}\right\|^{2}=A
$$

Proof: The trace of the UFBF matrix satisfies $\operatorname{tr}\left\{\mathbf{S}\left(e^{j 2 \pi \theta}\right)\right\}=\sum_{n=0}^{M-1} \sum_{k=0}^{N-1}\left|E_{k, n}\left(e^{j 2 \pi \theta}\right)\right|^{2}$, and, furthermore, $\operatorname{tr}\left\{\mathbf{S}\left(e^{j 2 \pi \theta}\right)\right\}=\sum_{n=0}^{M-1} \lambda_{n}(\theta)$ so that

$$
\sum_{n=0}^{M-1} \lambda_{n}(\theta)=\sum_{n=0}^{M-1} \sum_{k=0}^{N-1}\left|E_{k, n}\left(e^{j 2 \pi \theta}\right)\right|^{2} .
$$

From Corollary 5.1, we conclude that $M A \leq$ $\sum_{n=0}^{M-1} \lambda_{n}(\theta) \leq M B$, and with (11), $M A \leq \sum_{n=0}^{M-1} \sum_{k=0}^{N-1}$ $\left|E_{k, n}\left(e^{j 2 \pi \theta}\right)\right|^{2} \leq M B$. Integrating both sides of this inequality with respect to the frequency parameter $\theta$ and using $\sum_{n=0}^{M-1} \int_{0}^{1}\left|E_{k, n}\left(e^{j 2 \pi \theta}\right)\right|^{2} d \theta=\left\|h_{k}\right\|^{2}$ [39], we obtain (10).

If we normalize the $h_{k}[n]$ such that $\left\|h_{k}\right\|^{2}=1$ for $k=$ $0,1, \cdots, N-1$, then $\sum_{k=0}^{N-1}\left\|h_{k}\right\|^{2}=N$, and (10) yields the following inequality relating the frame bounds with the oversampling factor $N / M$

$$
A \leq \frac{N}{M} \leq B
$$

In particular, for a tight UFBF (corresponding to a paraunitary FB; see Section VI), it follows that the frame bounds are equal to the oversampling factor

$$
A=B=\frac{N}{M}
$$

\section{B. Frame Conditions}

We shall now derive conditions for an oversampled FB to provide a UFBF expansion in $l^{2}(\mathbb{Z})$. The next lemma discusses the existence of the upper frame bound $B<\infty$, which guarantees that the subband signals have finite energy if the input signal has finite energy.
Lemma 5.1: The analysis set $\left\{h_{k, m}[n]\right\}$ has an upper frame bound $B<\infty$, i.e.,

$$
\begin{gathered}
\sum_{k=0}^{N-1} \sum_{m=-\infty}^{\infty}\left|v_{k}[m]\right|^{2}=\sum_{k=0}^{N-1} \sum_{m=-\infty}^{\infty}\left|\left\langle x, h_{k, m}\right\rangle\right|^{2} \leq B\|x\|^{2} \\
\forall x[n] \in l^{2}(\mathbb{Z})
\end{gathered}
$$

if and only if the polyphase components $E_{k, n}\left(e^{j 2 \pi \theta}\right)$ are all bounded a.e., i.e., $\left|E_{k, n}\left(e^{j 2 \pi \theta}\right)\right| \leq K<\infty$ a.e. on $[0,1)$ for $k=0,1, \cdots, N-1, n=0,1, \cdots, M-1$.

Proof: Let $\left|E_{k, n}\left(e^{j 2 \pi \theta}\right)\right| \leq K<\infty$ a.e. It follows that the entries of the UFBF matrix $\mathbf{S}\left(e^{j 2 \pi \theta}\right)=$ $\mathbf{E}^{H}\left(e^{j 2 \pi \theta}\right) \mathbf{E}\left(e^{j 2 \pi \theta}\right)$ are bounded a.e., which implies that the $\lambda_{n}(\theta)$ are bounded a.e. Using Corollary 5.1, we conclude that $B<\infty$. We next prove the converse. Let $B=\operatorname{ess} \sup _{\theta \in[0,1), n=0,1, \cdots, M-1} \lambda_{n}(\theta)<\infty$. It follows that $\sum_{n=0}^{M-1} \lambda_{n}(\theta)$ is bounded a.e. With (11), this implies that the $E_{k, n}\left(e^{j 2 \pi \theta}\right)$ are bounded a.e.

We are now ready to formulate a necessary and sufficient condition for an FB to provide a UFBF expansion. The following theorem has previously been given for the important special case of FIR FB's in [12].

Theorem 5.1: An oversampled FB with bounded-input bounded-output (BIBO) stable ${ }^{7}$ analysis filters $h_{k}[n]$ provides a UFBF expansion in $l^{2}(\mathbb{Z})$, i.e., the analysis set $\left\{h_{k, m}[n]\right\}$ is a UFBF for $l^{2}(\mathbb{Z})$ if and only if the analysis polyphase matrix $\mathbf{E}(z)$ has full rank on the unit circle, ${ }^{8}$ i.e.,

$$
\operatorname{rank}\left\{\mathbf{E}\left(e^{j 2 \pi \theta}\right)\right\}=M \quad \text { for } 0 \leq \theta<1 .
$$

Proof: From $h_{k}[n] \in l^{1}(\mathbb{Z})$, it follows that the $E_{k, n}\left(e^{j 2 \pi \theta}\right)$ are bounded, and hence, we conclude from Lemma 5.1 that an upper frame bound $B<\infty$ exists. It remains to be shown that a full rank $\mathbf{E}\left(e^{j 2 \pi \theta}\right)$ is necessary and sufficient for the existence of a lower frame bound $A$. If $\mathbf{E}\left(e^{j 2 \pi \theta}\right)$ has full rank on $[0,1)$, then $\mathbf{S}\left(e^{j 2 \pi \theta}\right)=$ $\mathbf{E}^{H}\left(e^{j 2 \pi \theta}\right) \mathbf{E}\left(e^{j 2 \pi \theta}\right)$ has full rank on $[0,1)$, which means that $\lambda_{n}(\theta)>0$ for $0 \leq \theta<1$ and $n=0,1, \cdots, M-1$. From $h_{k}[n] \in l^{1}(\mathbb{Z})$, it follows that the $\lambda_{n}(\theta)$ are continuous functions of $\theta$, and therefore, we can conclude that ${ }^{9} A \equiv$ ess $\inf _{\theta \in[0,1), n=0,1} \cdots, M-1 \lambda_{n}(\theta)>0$. We next prove that, conversely, a full-rank $\mathbf{E}\left(e^{j 2 \pi \theta}\right)$ is necessary for the existence of $A>0$. Suppose that $\mathbf{E}\left(e^{j 2 \pi \theta}\right)$ does not have full rank on $[0,1)$. It follows that $\mathbf{S}\left(e^{j 2 \pi \theta}\right)$ does not have full rank on $[0,1)$. This implies that there is at least one eigenvalue with $\lambda_{n}(\theta)=0$ on a measurable set with positive measure. Hence, using Corollary 5.1, we conclude that $A=0$.

Alternatively, it can be shown that an FB corresponds to a UFBF for $l^{2}(\mathbb{Z})$ if $\mathbf{E}\left(e^{j 2 \pi \theta}\right)$ has full rank for $0 \leq \theta<1$, and the $E_{k, n}\left(e^{j 2 \pi \theta}\right)$ are continuous and bounded functions of $\theta$. Yet another condition, which is phrased in terms of the eigenvalues of the UFBF matrix $\mathbf{S}\left(e^{j 2 \pi \theta}\right)$, follows easily from Corollary 3.1:

${ }^{7}$ BIBO stability means that $h_{k}[n] \in l^{1}(\mathbb{Z})$, i.e., $\sum_{n=-\infty}^{\infty}\left|h_{k}[n]\right|<\infty$ for $k=0,1, \cdots, N-1$.

${ }^{8}$ We emphasize that $\mathbf{E}(z)$ is here required to have full rank everywhere on the unit circle. In contrast, the completeness condition in Theorem 4.2 merely required $\mathbf{E}(z)$ to have full rank a.e. on the unit circle.

${ }^{9}$ For a continuous function, the essential infimum is the infimum (this is, however, not relevant to this proof) 


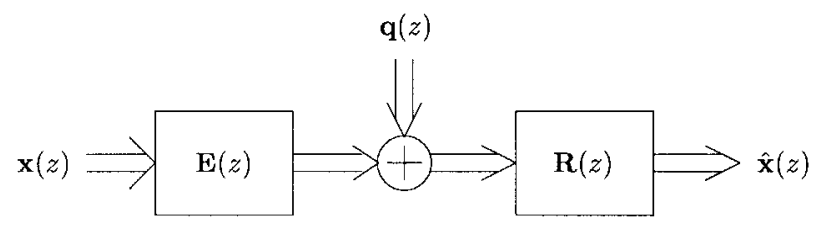

Fig. 2. Adding noise to the subband signals.

Corollary 5.3: An oversampled FB provides a UFBF expansion in $l^{2}(\mathbb{Z})$ if and only if the eigenvalues $\lambda_{n}(\theta)$ of the UFBF matrix $\mathbf{S}\left(e^{j 2 \pi \theta}\right)=\mathbf{E}^{H}\left(e^{j 2 \pi \theta}\right) \mathbf{E}\left(e^{j 2 \pi \theta}\right)$ satisfy

and

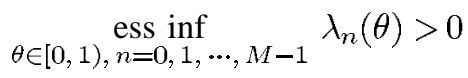

$$
\operatorname{ess~sup}_{\theta \in[0,1), n=0,1, \ldots, M-1} \lambda_{n}(\theta)<\infty .
$$

Proof: It is known [28], [30], [33] that $\left\{h_{k, m}[n]\right\}$ is a frame if and only if ess inf $\lambda>0$ and ess sup $\lambda<\infty$, where $\{\lambda\}$ is the set of all eigenvalues of the frame operator $S$. Due to Corollary 3.1, the eigenvalues of $S$ equal the eigenvalues $\lambda_{n}(\theta)$ of $\mathbf{S}\left(e^{j 2 \pi \theta}\right)$, which completes the proof.

Using the fact that FIR filters are inherently BIBO stable and, thus, one of the conditions of Theorem 5.1 is here always satisfied, it follows that an oversampled FB with FIR analysis filters provides a UFBF expansion in $l^{2}(\mathbb{Z})$ if and only if the analysis polyphase matrix $\mathbf{E}(z)$ has full rank on the unit circle, i.e., $\operatorname{rank}\left\{\mathbf{E}\left(e^{j 2 \pi \theta}\right)\right\}=M$ for $0 \leq \theta<1$. This result has been previously reported by Cvetković and Vetterli [12].

\section{Sensitivity Analysis}

Important numerical properties of the UFBF $\left\{h_{k, m}[n]\right\}$ and, thus, of the associated FB as well, are determined by its frame bounds $A$ and $B$ [28]. Let us investigate the sensitivity of oversampled FB's to (quantization) noise $q_{k}[\mathrm{~m}]$ added to the subband signals $v_{k}[m]=\left\langle x, h_{k, m}\right\rangle(k=0,1, \cdots, N-1)$. We collect the noise signals $q_{k}[\mathrm{~m}]$ in the $N$-dimensional vector noise process $\mathbf{q}[\mathrm{m}]$ that is assumed to be wide-sense stationary and zero-mean. The $N \times N$ power spectral matrix of $\mathbf{q}[\mathrm{m}]$ is defined as $\mathbf{S}_{q}(z)=\sum_{l=-\infty}^{\infty} \mathbf{C}_{q}[l] z^{-l}$ with the autocorrelation matrix $\mathbf{C}_{q}[l]=\mathcal{E}\left\{\mathbf{q}[m] \mathbf{q}^{H}[m-l]\right\}$, where $\mathcal{E}$ denotes the expectation operator [1].

It is convenient to redraw the FB in the "polyphase domain," as shown in Fig. 2 [1]. Here, the polyphase vectors $\mathbf{x}(z)$ and $\hat{\mathbf{x}}(z)$ are defined as in Section III-B, and $\mathbf{q}(z)=$ $\sum_{m=-\infty}^{\infty} \mathbf{q}[m] z^{-m}$ is the $z$-transform of the noise $\mathbf{q}[m]$. Assuming a PR FB, we have (see Fig. 2) $\hat{\mathrm{x}}(z)=\mathrm{x}(z)+$ $\mathbf{R}(z) \mathbf{q}(z)$ so that the reconstruction error $e[n]=\hat{x}[n]-x[n]$ is given by

$$
\mathbf{e}(z)=\hat{\mathbf{x}}(z)-\mathbf{x}(z)=\mathbf{R}(z) \mathbf{q}(z) .
$$

The reconstruction error $e[n]$ is again wide-sense stationary and zero-mean, with $M \times M$ power spectral matrix [1]

$$
\mathbf{S}_{e}(z)=\mathbf{R}(z) \mathbf{S}_{q}(z) \tilde{\mathbf{R}}(z)
$$

and variance

$$
\sigma_{e}^{2}=\frac{1}{M} \int_{0}^{1} \operatorname{tr}\left\{\mathbf{S}_{e}\left(e^{j 2 \pi \theta}\right)\right\} d \theta
$$

where tr denotes the trace. With the idealizing assumption that the noise signals $q_{k}[\mathrm{~m}]$ are uncorrelated and white with identical variances $\sigma_{q}^{2}=\mathcal{E}\left\{\left|q_{k}[m]\right|^{2}\right\}$, i.e., $\mathbf{C}_{q}[l]=\sigma_{q}^{2} \mathbf{I}_{N} \delta[l]$ and $\mathbf{S}_{q}(z)=\sigma_{q}^{2} \mathbf{I}_{N}$ [1], the error variance becomes

$$
\sigma_{e}^{2}=\frac{\sigma_{q}^{2}}{M} \int_{0}^{1} \operatorname{tr}\left\{\mathbf{R}\left(e^{j 2 \pi \theta}\right) \mathbf{R}^{H}\left(e^{j 2 \pi \theta}\right)\right\} d \theta .
$$

We will now restrict our attention to the PR synthesis FB corresponding to the dual frame, i.e., $\mathbf{R}(z)=\hat{\mathbf{R}}(z)$. With $\operatorname{tr}\left\{\hat{\mathbf{R}}\left(e^{j 2 \pi \theta}\right) \hat{\mathbf{R}}^{H}\left(e^{j 2 \pi \theta}\right)\right\}=\sum_{n=0}^{M-1} \lambda_{n}^{\prime}(\theta)$, where $\lambda_{n}^{\prime}(\theta)$ denotes the eigenvalues of the inverse UFBF matrix $\mathrm{S}^{-1}\left(e^{j 2 \pi \theta}\right)$, and using $A^{\prime} \leq \lambda_{n}^{\prime}(\theta) \leq B^{\prime}$ (see Corollary 5.1) or equivalently $1 / B \leq \lambda_{n}^{\prime}(\theta) \leq 1 / A$, we obtain $M / B \leq \operatorname{tr}\left\{\hat{\mathbf{R}}\left(e^{j 2 \pi \theta}\right) \mathbf{R}^{H}\left(e^{j 2 \pi \theta}\right)\right\} \leq M / A$. Inserting this in (14), we further obtain

$$
\frac{1}{B} \leq \frac{\sigma_{e}^{2}}{\sigma_{q}^{2}} \leq \frac{1}{A}
$$

i.e., the reconstruction error variance $\sigma_{e}^{2}$ is bounded in terms of the frame bounds $A, B$. Let us assume normalized analysis filters, i.e., $\left\|h_{k}\right\|=1$ for $k=0,1, \cdots, N-1$. Then, (12) yields $1 / B \leq 1 / K \leq 1 / A$, where $K=N / M$ is the oversampling factor. Hence, for $A \approx B$ or equivalently $B / A \approx 1$ (snug frame), (15) implies

$$
\frac{\sigma_{e}^{2}}{\sigma_{q}^{2}} \approx \frac{1}{K} \quad \text { with } K=\frac{N}{M}
$$

which means that small perturbations of the subband signals yield small reconstruction error. We note that the design of FB's with $B / A \approx 1$ (and additional desirable properties such as good frequency selectivity) is easier for larger oversampling factor (see Section VIII).

For a paraunitary FB with $\left\|h_{k}\right\|=1$, we have $A=B=K$ [see (13)], and hence, (15) becomes

$$
\frac{\sigma_{e}^{2}}{\sigma_{q}^{2}}=\frac{1}{K} .
$$

Thus, in the paraunitary case, the reconstruction error variance is inversely proportional to the oversampling factor $K$, which means that more oversampling entails more noise reduction. Such a " $1 / K$ behavior" of the reconstruction error variance has previously been observed for oversampled A/D conversion [50], for tight frames in finite dimensional spaces [28], [51], and for reconstruction from a finite set of Weyl-Heisenberg (Gabor) or wavelet coefficients [28], [37]. Recently, under additional conditions, a $1 / K^{2}$ behavior has been demonstrated for Weyl-Heisenberg frames [20], [37], [52].

\section{Approximative Construction of the Synthesis Filter Bank}

The calculation of the minimum norm, PR synthesis FB (para-pseudoinverse) $\hat{\mathbf{R}}(z)=[\tilde{\mathbf{E}}(z) \mathbf{E}(z)]^{-1} \tilde{\mathbf{E}}(z)$ requires the inversion of the matrix $\mathbf{E}(z) \mathbf{E}(z)$, which is a cumbersome task in general. If the FB corresponds to a UFBF, then an approximative calculation of the minimum norm synthesis FB (which is analogous to the approximation of dual frames described in [33]) can be based on a series expansion of 
$\mathbf{S}^{-1}(z)=[\tilde{\mathbf{E}}(z) \mathbf{E}(z)]^{-1}$. Indeed, applying the Neumann series expansion [44] to the matrix $[\tilde{\mathbf{E}}(z) \mathbf{E}(z)]^{-1}$, the minimum norm synthesis FB is expressed as

$$
\hat{\mathbf{R}}(z)=\frac{2}{A+B}\left[\sum_{i=0}^{\infty}\left(\mathbf{I}_{M}-\frac{2}{A+B} \tilde{\mathbf{E}}(z) \mathbf{E}(z)\right)^{i}\right] \tilde{\mathbf{E}}(z) .
$$

The convergence of this series expansion follows from frame theory [28] using the correspondence between the frame operator $S$ and the UFBF matrix $\mathbf{S}(z)=\tilde{\mathbf{E}}(z) \mathbf{E}(z)$; it will be faster for snugger frames, i.e., for closer frame bounds $A, B$.

By truncating the expansion (16), the synthesis FB can be approximated with arbitrary accuracy. Estimates of the resulting reconstruction error are available [28]. We shall here restrict our attention to the zero-order approximation of $\hat{\mathbf{R}}(z)$ obtained by retaining only the $i=0$ term in (16)

$$
\hat{\mathbf{R}}_{0}(z)=\frac{2}{A+B} \tilde{\mathbf{E}}(z)
$$

which corresponds to an approximation of the minimum norm synthesis filters $\hat{f}_{k}[n]$ as

$$
\hat{f}_{k ; 0}[n]=\frac{2}{A+B} h_{k}^{*}[-n]
$$

The reconstruction error resulting from this approximation can be bounded in terms of the frame bounds $A$ and $B$. With $\hat{x}_{0}[n]$ denoting the signal reconstructed using the above "zero-order synthesis FB" $\left\{\hat{f}_{k ; 0}[n]\right\}$, we have the error bound [33]

$$
\left\|\hat{x}_{0}-x\right\| \leq \frac{B / A-1}{A / B+1}\|x\| .
$$

We see that the reconstruction error is small for $B / A \approx 1$, i.e., when the underlying UFBF is snug. Thus, in the snug case, the synthesis impulse responses $\hat{f}_{k ; 0}[n]$ are a good approximation to the true minimum norm, PR impulse responses $\hat{f}_{k}[n]$, in the sense that the resulting reconstruction error $\left\|\hat{x}_{0}-x\right\|$ is small. In the tight case where $B / A=1$, the reconstruction error becomes zero altogether, and indeed, the approximation is here exact as $\hat{f}_{k ; 0}[n]=\hat{f}_{k}[n]=(1 / A) h_{k}^{*}[-n]$.

Besides the trivial zero-order approximation discussed above, the series expansion (16) also allows the iterative calculation of the minimum norm synthesis filters $\hat{f}_{k}[n]$. Sophisticated algorithms for this iteration have been proposed in [53] in a frame-theoretic setting. Using the correspondences established further above, the reformulation of these algorithms in the present FB framework is straightforward.

\section{OVERSAMPLED PARAUNITARY FiLTER BANKS AND TIGHT FRAMES}

In this section, we show that oversampled paraunitary FB's provide tight UFBF expansions in $l^{2}(\mathbb{Z})$, and we discuss a frame-theoretic method for constructing paraunitary FB's from given nonparaunitary FB's.

\section{A. Equivalence of Oversampled Paraunitary Filter Banks and Tight Frames}

The analysis UFBF $\left\{h_{k, m}[n]\right\}$ is tight if $A=B$. From frame theory, we know that here, $S=A \boldsymbol{I}$ [28]. With (4), this implies that the frame-theoretic (i.e., minimum norm) solution for the PR synthesis FB is

$$
\hat{f}_{k}[n]=\frac{1}{A} h_{k}^{*}[-n]
$$

or equivalently $\hat{\mathbf{R}}(z)=(1 / A) \tilde{\mathbf{E}}(z)$. This is precisely the relation between the synthesis and analysis filters in a paraunitary FB [1]. In fact, we can formulate the following theorem that extends a result previously reported in [12] for the FIR case and in [54] for the case of complex modulated (DFT) FB's.

Theorem 6.1: An oversampled FB provides a tight UFBF expansion in $l^{2}(\mathbb{Z})$ if and only if it is paraunitary, i.e.,

$$
\mathbf{S}(z)=\tilde{\mathbf{E}}(z) \mathbf{E}(z) \equiv A \mathbf{I}_{M} .
$$

The frame bound is given by $A=S_{n, n}(z)=$ $\sum_{k=0}^{N-1} \tilde{E}_{k, n}(z) E_{k, n}(z)$.

Proof: From $\mathbf{S}(z)=A \mathbf{I}_{M}$, it follows with (6) that $\mathbf{y}(z)=A \mathbf{x}(z)$, which implies $y[n]=A x[n]$. Hence, comparing with $y[n]=(\boldsymbol{S} x)[n]$, we conclude that $\boldsymbol{S}=A \boldsymbol{I}$, i.e., $\left\{h_{k, m}[n]\right\}$ is a tight UFBF with frame bound $A$. The converse statement is proven by reversing this line of reasoning. Combining $S_{n, n}(z)=\sum_{k=0}^{N-1} \tilde{E}_{k, n}(z) E_{k, n}(z)$ and $\mathbf{S}(z)=A \mathbf{I}_{M}$, it follows that $A=S_{n, n}(z)=\sum_{k=0}^{N-1} \tilde{E}_{k, n}(z) E_{k, n}(z)$.

Paraunitary FB's are also known as orthogonal FB's. However, the name "orthogonal" is justified only in the critical case since critically sampled paraunitary FB's provide decompositions into orthogonal UFBF's. In the oversampled case, paraunitary FB's correspond to UFBF's that are tight but not orthogonal.

\section{B. Construction of Paraunitary FB's}

We next describe a procedure for the derivation of a paraunitary FB from a given nonparaunitary FB. From frame theory, we know that application of the positive definite operator square root $S^{-1 / 2}$ to each of the frame functions $h_{k, m}[n]$ produces a tight frame [28], [30]. Using the correspondence between the frame operator $S$ and the UFBF matrix $\mathbf{S}(z)=$ $\tilde{\mathbf{E}}(z) \mathbf{E}(z)$, the following result is obtained.

Theorem 6.2: Consider an FB corresponding to a UFBF expansion, and let $\mathbf{P}(z)$ be an invertible, para-Hermitian, ${ }^{10}$ $M \times M$ matrix such that $\mathbf{P}^{2}(z)=\tilde{\mathbf{E}}(z) \mathbf{E}(z)$, where $\mathbf{E}(z)$ is the FB's analysis polyphase matrix. Then, the FB with analysis polyphase matrix

$$
\mathbf{E}^{(p)}(z)=\mathbf{E}(z) \mathbf{P}^{-1}(z)
$$

is paraunitary with frame bound $A=1$, i.e., $\mathbf{S}^{(p)}(z)=$ $\tilde{\mathbf{E}}^{(p)}(z) \mathbf{E}^{(p)}(z) \equiv \mathbf{I}_{M}$. If, moreover, in the case of critical sampling the original FB is biorthogonal, then the FB with analysis polyphase matrix $\mathbf{E}^{(p)}(z)$ is orthogonal.

\footnotetext{
${ }^{10} \mathrm{~A}$ matrix $\mathbf{P}(z)$ is said to be para-Hermitian if $\tilde{\mathbf{P}}(z)=\mathbf{P}(z)$ [55].
} 
Proof: We have $\mathbf{S}^{(p)}(z)=\tilde{\mathbf{E}}^{(p)}(z) \mathbf{E}^{(p)}(z)=$ $\tilde{\mathbf{P}}^{-1}(z) \tilde{\mathbf{E}}(z) \mathbf{E}(z) \mathbf{P}^{-1}(z)$. Inserting $\tilde{\mathbf{E}}(z) \mathbf{E}(z)=\mathbf{P}^{2}(z)$ in the right-hand side and using $\tilde{\mathbf{P}}(z)=\mathbf{P}(z)$, it follows that $\tilde{\mathbf{E}}^{(p)}(z) \mathbf{E}^{(p)}(z)=\mathbf{I}_{M}$, which shows that the FB with polyphase matrix $\mathbf{E}^{(p)}(z)$ is paraunitary with frame bound $A=1$.

From frame theory [28], [30], we know that by applying the procedure described above to an exact frame (corresponding to a biorthogonal FB), an orthogonal function set (corresponding to an orthogonal FB) is obtained. Note that a UFBF can be exact only in the case of critical sampling.

The matrix $\mathrm{P}^{-1}(z)$ can be calculated by performing a factorization of $\mathrm{P}^{-2}(z)=\hat{\mathbf{R}}(z) \tilde{\hat{\mathbf{R}}}(z)$. A detailed study of such a factorization problem for both polynomial and rational matrices is given in [56]. Alternatively, the approximative calculation of $\mathrm{P}^{-1}(z)$ can be based on a series expansion similar to (16): Using the correspondence between the frame operator $\boldsymbol{S}$ and the UFBF matrix $\mathbf{S}(z)=\tilde{\mathbf{E}}(z) \mathbf{E}(z)$, we have [57]

$$
\begin{aligned}
\mathbf{P}^{-1}(z)= & \sqrt{\frac{2}{A+B}} \sum_{i=0}^{\infty} \frac{(2 i) !}{2^{2 i}(i !)^{2}} \\
& \cdot\left(\mathbf{I}_{M}-\frac{2}{A+B} \tilde{\mathbf{E}}(z) \mathbf{E}(z)\right)^{i} .
\end{aligned}
$$

\section{SPECIAL CASES}

In this section, we discuss FB's whose frame operator becomes a simple multiplication operator in the polyphase domain or in the frequency domain, i.e., the polyphase representation or the Fourier transform "diagonalizes" the frame operator. This class of FB's comprises integer oversampled or critically sampled DFT FB's, nondecimated FB's, and bandlimited FB's. We shall see that the calculation of the synthesis FB, of the frame bounds, and of paraunitary FB's is drastically simplified in these cases.

\section{A. Diagonality in the Polyphase Domain}

According to Lemma 3.1, the frame operator $S$ is represented in the polyphase domain by the UFBF matrix $\mathbf{S}(z)$. Consequently, a FB corresponding to a UFBF is "diagonal in the polyphase domain" if the UFBF matrix is a diagonal matrix

$$
\begin{aligned}
& \mathbf{S}(z)=\tilde{\mathbf{E}}(z) \mathbf{E}(z)=\operatorname{diag}\left\{S_{n, n}(z)\right\}_{n=0}^{M-1} \\
& \text { with } S_{n, n}(z)=\sum_{k=0}^{N-1} \tilde{E}_{k, n}(z) E_{k, n}(z) .
\end{aligned}
$$

It follows from (8) that the polyphase matrix of the minimum norm synthesis FB is given by

$$
\begin{aligned}
\hat{\mathbf{R}}(z) & =\operatorname{diag}\left\{\frac{1}{S_{n, n}(z)}\right\}_{n=0}^{M-1} \tilde{\mathbf{E}}(z) \\
\hat{R}_{k, n}(z) & =\frac{\tilde{E}_{k, n}(z)}{S_{n, n}(z)} .
\end{aligned}
$$

We can see that the calculation of the minimum norm synthesis $\mathrm{FB}$, which in general requires the inversion of the UFBF matrix $\mathbf{S}(z)=\tilde{\mathbf{E}}(z) \mathbf{E}(z)$, reduces to simple divisions in the polyphase domain.

Using the fact that the eigenvalues of the diagonal matrix $\mathbf{S}\left(e^{j 2 \pi \theta}\right)=\mathbf{E}^{H}\left(e^{j 2 \pi \theta}\right) \mathbf{E}\left(e^{j 2 \pi \theta}\right)$ are given by

$$
\lambda_{n}(\theta)=S_{n, n}\left(e^{j 2 \pi \theta}\right)=\sum_{k=0}^{N-1}\left|E_{k, n}\left(e^{j 2 \pi \theta}\right)\right|^{2}
$$

it follows from Corollary 5.3 that the FB corresponds to a UFBF if and only if

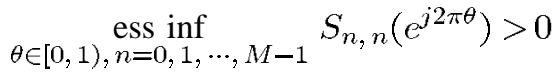

and

$$
\underset{\theta \in[0,1), n=0,1, \cdots, M-1}{\operatorname{ess} \sup } S_{n, n}\left(e^{j 2 \pi \theta}\right)<\infty
$$

and according to Corollary 5.1, the frame bounds are given by

$$
\begin{aligned}
& A=\underset{\theta \in[0,1), n=0,1, \cdots, M-1}{\operatorname{ess} \inf _{n, n}\left(e^{j 2 \pi \theta}\right),} \\
& B=\underset{\theta \in[0,1), n=0,1, \cdots, M-1}{\operatorname{ess} \sup _{n, n}\left(e^{j 2 \pi \theta}\right) .}
\end{aligned}
$$

In particular, the FB is paraunitary with frame bound $A$ if and only if

$$
\begin{aligned}
S_{n, n}(z)= & \sum_{k=0}^{N-1} \tilde{E}_{k, n}(z) E_{k, n}(z) \equiv A \\
& \text { for } n=0,1, \cdots, M-1 .
\end{aligned}
$$

The construction of paraunitary FB's from nonparaunitary FB's (see Theorem 6.2) simplifies as well. Consider an FB with analysis polyphase components $E_{k, n}(z)$, and define $P_{n}(z)$ by $P_{n}^{2}(z)=S_{n, n}(z)$ with $\tilde{P}_{n}(z)=P_{n}(z)$. Then, the FB with analysis polyphase components

$$
E_{k, n}^{(p)}(z)=\frac{E_{k, n}(z)}{P_{n}(z)}
$$

is paraunitary with $A=1$, i.e., $\tilde{\mathbf{E}}^{(p)}(z) \mathbf{E}^{(p)}(z) \equiv \mathbf{I}_{M}$. Thus, the matrix factorization $\tilde{\mathbf{E}}(z) \mathbf{E}(z)=\mathbf{P}^{2}(z)$ reduces to a factorization of polynomials in $z$ (in the FIR case) or rational functions in $z$ (in the IIR case).

Integer oversampled or critically sampled DFT FB's [4], [6], [7], [11], [16], [24], [39], [54], [58] are an important example of FB's that are diagonal in the polyphase domain. The corresponding UFBF type is the important class of Weyl-Heisenberg frames [16], [25], [28], [30], [33], [34], [39], [59], [60]. In a DFT FB, the analysis filters are modulated versions of a single analysis prototype filter $h[n]$, i.e., $h_{k}[n]=h[n] W_{N}^{-k n n}$ with $W_{N}=e^{-j 2 \pi / N}$. The minimum norm synthesis FB has the same structure, i.e., $\hat{f}_{k}[n]=\hat{f}[n] W_{N}^{-k n}$ [16]. The polyphase components are

$$
E_{k, n}(z)=W_{N}^{k n} E_{n}\left(z W_{N}^{M k}\right)
$$

with

$$
E_{n}(z)=\sum_{m=-\infty}^{\infty} h[m M-n] z^{-m}, n=0,1, \cdots, M-1
$$


and

$$
\hat{R}_{k, n}(z)=W_{N}^{-k n} \hat{R}_{n}\left(z W_{N}^{M k}\right)
$$

with

$$
\hat{R}_{n}(z)=\sum_{m=-\infty}^{\infty} \hat{f}[m M+n] z^{-m}, n=0,1, \cdots, M-1 .
$$

In the important cases of integer oversampling $(N=K M$ with $K \in \mathbb{N}, K \geq 2$ ) and critical sampling ( $N=M$, i.e., $K=1$ ), the DFT FB is diagonal in the polyphase domain with

$$
S_{n, n}(z)=M \sum_{l=0}^{K-1} \tilde{E}_{n}\left(z W_{K}^{l}\right) E_{n}\left(z W_{K}^{l}\right)
$$

and

$$
\lambda_{n}(\theta)=S_{n, n}\left(e^{j 2 \pi \theta}\right) M \sum_{l=0}^{K-1}\left|E_{n}\left(e^{j 2 \pi(\theta-(l / K))}\right)\right|^{2} .
$$

Hence, all results presented further above for diagonal FB's apply to integer oversampled or critically sampled DFT FB's. In particular, (19) simplifies to

$$
\hat{R}_{n}(z)=\frac{\tilde{E}_{n}(z)}{S_{n, n}(z)} .
$$

In [5] and [6], it has been shown that for critical sampling, a DFT FB with PR and FIR filters in both the analysis and the synthesis section is possible only if all the polyphase filters are pure delays. This leads to filters with poor frequency selectivity. In the oversampled case, this restriction is relaxed. For $K=2$, for example, a paraunitary DFT FB with FIR filters can be constructed by using polyphase filters that satisfy the power symmetry conditions [1]

$$
\begin{aligned}
\tilde{E}_{n}(z) E_{n}(z)+\tilde{E}_{n}(-z) E_{n}(-z)=\frac{A}{M} \\
\text { for } n=0,1, \cdots, M-1 .
\end{aligned}
$$

It is well known that this can be achieved with FIR filters corresponding to nontrivial polyphase filters [1]. Oversampled DFT FB's with good frequency localization have also been constructed in [11].

\section{B. Diagonality in the Frequency Domain}

An FB corresponding to a UFBF is "diagonal in the frequency domain" if its frame operator is a simple multiplication operator in the frequency domain ( $z$-transform domain). With $y[n]=(\boldsymbol{S} x)[n]$, this means

$$
Y(z)=G(z) X(z) \quad \text { with } G(z)=\frac{1}{M} \sum_{k=0}^{N-1} H_{k}(z) \tilde{H}_{k}(z)
$$

where $X(z), Y(z)$, and $H_{k}(z)$ denote the $z$-transforms of $x[n]$, $y[n]$, and $h_{k}[n]$, respectively. The eigenvalues of the UFBF matrix $\mathbf{S}\left(e^{j 2 \pi \theta}\right)$ are here given by

$$
\lambda(\theta)=G\left(e^{j 2 \pi \theta}\right)=\frac{1}{M} \sum_{k=0}^{N-1}\left|H_{k}\left(e^{j 2 \pi \theta}\right)\right|^{2} .
$$

Two important classes of FB's that are diagonal in the frequency domain are nondecimated FB's, i.e., FB's with no decimation in the subbands ${ }^{11}$ or $M=1$, and bandlimited FB's, i.e., FB's whose analysis filters have bandwidth $\leq 1 / M$. We note that nondecimated FIR FB's have been studied previously by Cvetković and Vetterli in [12].

With (20), it follows that the $z$-transforms of the minimum norm synthesis filters are obtained as

$$
\hat{F}_{k}(z)=\frac{\tilde{H}_{k}(z)}{G(z)} .
$$

Furthermore, (21) implies that the frame condition can be reformulated as

$$
\underset{\theta \in[0,1)}{\operatorname{ess} \inf } G\left(e^{j 2 \pi \theta}\right)>0 \quad \text { and } \quad \underset{\theta \in[0,1)}{\operatorname{ess} \sup } G\left(e^{j 2 \pi \theta}\right)<\infty .
$$

With $G\left(e^{j 2 \pi \theta}\right)=(1 / M) \sum_{k=0}^{N-1}\left|H_{k}\left(e^{j 2 \pi \theta}\right)\right|^{2}$, the lower bound means that the set of analysis filters has to "cover" the entire frequency interval $[0,1)$. This condition is satisfied if and only if the analysis filters have no zeros in common on the unit circle. The upper bound is automatically satisfied for BIBO stable filters, i.e., $h_{k}[n] \in l^{1}(\mathbb{Z})$ for $k=0,1, \cdots, N-1$. The frame bounds are given by

$$
A=\underset{\theta \in[0,1)}{\operatorname{ess} \inf } G\left(e^{j 2 \pi \theta}\right), \quad B=\underset{\theta \in[0,1)}{\operatorname{ess} \sup } G\left(e^{j 2 \pi \theta}\right) .
$$

Paraunitarity with frame bound $A$ implies

$$
G(z)=\frac{1}{M} \sum_{k=0}^{N-1} H_{k}(z) \tilde{H}_{k}(z) \equiv A
$$

which means that the analysis filters $H_{k}(z)$ are power complementary [1]. Paraunitary FB's with frame bound $A=1$ can be constructed by solving the factorization $P^{2}(z)=G(z)$ with $\tilde{P}(z)=P(z)$; the paraunitary analysis filters are then given by

$$
H_{k}^{(p)}(z)=\frac{H_{k}(z)}{P(z)} .
$$

\section{Simulation Results}

We now present simulation results demonstrating the importance of snug frames $(B / A \approx 1)$ and the benefits of oversampling. We consider a DFT FB (see Section VII-A) with $N=64$ channels and a 192-tap lowpass analysis prototype filter $h[n]$. The simulation results were obtained by performing all calculations within the framework of cyclic DFT FB's (cyclic Weyl-Heisenberg frames) [39] with period 192. The dual windows and the frame bounds we obtained are, hence, approximations to the true (i.e., noncyclic) dual windows and frame bounds.

The analysis prototype filter $h[n]$ is depicted in Fig. 3(a). Fig. 3(b)-(d) shows the minimum norm synthesis prototype filters for oversampling by the factors 2,4 , and 8 , respectively. The frame bound ratio $B / A$ was estimated as $33.258,2.260$,

\footnotetext{
${ }^{11}$ We note that nondecimated FB's are also trivially diagonal in the polyphase domain.
} 


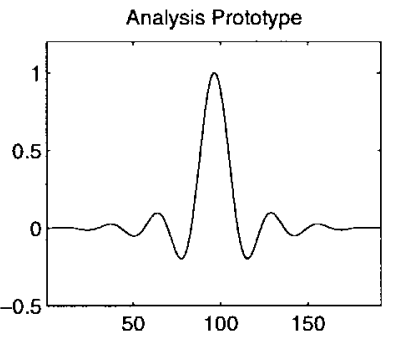

(a)

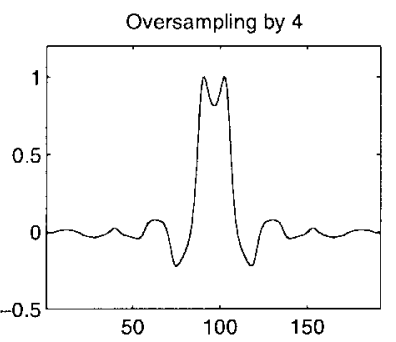

(c)

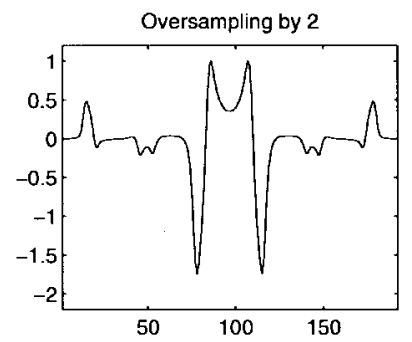

(b)

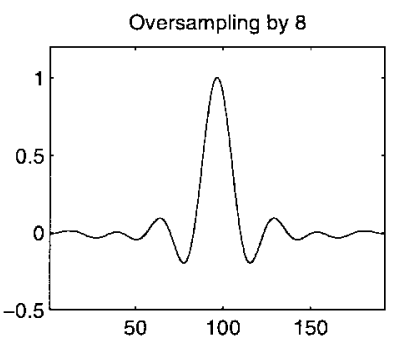

(d)
Fig. 3. Comparison of analysis and minimum norm synthesis prototype filters for various frame bound ratios and oversampling factors. (a) Analysis prototype $h[n]$. (b)-(d) Minimum norm synthesis prototype $f[n]$ for (b) oversampling by 2 (resulting in $B / A=33.258$ ), (c) oversampling by 4 ( $B / A=2.260)$, and (d) oversampling by $8(B / A=1.107)$.

TABLE I

Frame Bound Ratio $B / A$ as a Function OF THE Oversampling Factor $N / M$

\begin{tabular}{r|r||r|r}
\hline$N$ & \multicolumn{1}{|r||}{$M$} & $N / M$ & $B / A$ \\
\hline 64 & 64 & 1.00 & $1.257 \cdot 10^{4}$ \\
64 & 48 & 1.33 & 144.186 \\
64 & 32 & 2.00 & 33.258 \\
64 & 24 & 2.66 & 28.411 \\
64 & 16 & 4.00 & 2.260 \\
64 & 12 & 5.33 & 1.107 \\
64 & 8 & 8.00 & 1.107 \\
64 & 6 & 10.66 & 1.102 \\
64 & 4 & 16.00 & 1.102 \\
64 & 3 & 21.33 & 1.102 \\
64 & 2 & 32.00 & 1.102 \\
64 & 1 & 64.00 & 1.102 \\
\hline
\end{tabular}

and 1.107 , respectively. Thus, more oversampling is seen to result in snugger frames.

It is furthermore seen that for snugger frames (i.e., more oversampling), the minimum norm synthesis prototype is increasingly similar to the analysis prototype. Approximating the synthesis FB using the zero-order approximation in (17) (i.e., essentially using the analysis $\mathrm{FB}$ as synthesis $\mathrm{FB}$ ) resulted in the following upper bounds $b=(B / A-1) /(A / B+1)$ on the normalized reconstruction error $\left\|\hat{x}_{0}-x\right\| /\|x\|$ [see (18)]:

- $b=31.315$ for oversampling by 2 ;

- $b=0.874$ for oversampling by 4 ;

- $b=0.056$ for oversampling by 8 .

Thus, the reconstruction error can be expected to be negligible if the oversampling factor is sufficiently large.

For the DFT FB analysis prototype in Fig. 3(a), Table I shows the frame bound ratio $B / A$ as a function of the oversampling factor $N / M$ (note that $N / M=1$ means critical sampling). We see that $B / A$ is closer to 1 (i.e., the frame is snugger) for increasing oversampling factor; for $N / M \geq 5.33$, the FB is nearly paraunitary.

We caution, however, that if the prototype $h[n]$ does not "match" the time-frequency grid determined by the parameters $N$ and $M$, it is not guaranteed that the frame bound ratio will improve for increasing oversampling factor [61]. Furthermore, we note that paraunitary FB's (corresponding to tight frames, i.e., $A=B$ ) can, of course, also be constructed in the case of critical sampling. However, in the oversampled case, the filters of a paraunitary FB tend to have desirable properties (such as improved frequency selectivity). This is due to the fact that in the design of an oversampled PR FB, there are fewer side constraints to be satisfied than in the case of critical sampling.

\section{CONCLUSION}

We have shown that the theory of frames is a powerful vehicle for the analysis and design of oversampled filter banks (FB's). A key result on which most of our theory was based is the fact that the polyphase matrices provide matrix representations of the frame operator. We demonstrated that the frame bounds characterize important numerical properties of FB's and that they can be obtained by an eigenanalysis of the polyphase matrices. For a given oversampled analysis FB, we provided a compact and useful parameterization of all synthesis FB's providing perfect reconstruction, and we discussed the perfect reconstruction synthesis FB with minimum norm (i.e., the particular synthesis FB obtained from frame theory) and its approximative construction. We formulated conditions for an oversampled FB to provide a frame decomposition. We also proposed a new method for constructing paraunitary FB's from given nonparaunitary FB's. Finally, we presented simulation results demonstrating the benefits of snug frames and oversampling.

\section{ACKNOWLEDGMENT}

The authors would like to thank the anonymous reviewers for valuable comments that have led to improvements in this paper.

\section{REFERENCES}

[1] P. P. Vaidyanathan, Multirate Systems and Filter Banks. Englewood Cliffs, NJ: Prentice-Hall, 1993.

[2] M. Vetterli and J. Kovačević, Wavelets and Subband Coding. Englewood Cliffs, NJ: Prentice-Hall, 1995.

[3] A. N. Akansu and R. A. Haddad, Multiresolution Signal Decomposition. New York: Academic, 1992.

[4] R. E. Crochiere and L. R. Rabiner, Multirate Digital Signal Processing. Englewood Cliffs, NJ: Prentice-Hall, 1983.

[5] M. Vetterli, "A theory of multirate filter banks," IEEE Trans. Acoust., Speech, Signal Processing vol. ASSP-35, pp. 356-372, Mar. 1987.

[6] K. Swaminathan and P. P. Vaidyanathan, "Theory and design of uniform DFT, parallel, quadrature mirror filter banks," IEEE Trans. Circuits Syst., vol. CAS-33, pp. 1170-1191, Nov. 1986.

[7] M. J. T. Smith and T. P. Barnwell, "A new filter bank theory for time-frequency representation," IEEE Trans. Acoust., Speech, Signal Processing, vol. ASSP-35, pp. 314-327, Mar. 1987.

[8] T. Chen and P. P. Vaidyanathan, "Vector space framework for unification of one- and multidimensional filter bank theory," IEEE Trans. Signal Processing, vol. 42, pp. 2006-2021, Aug. 1994.

[9] S. G. Mallat, A Wavelet Tour of Signal Processing. San Diego, CA: Academic, 1998. 
[10] Y. Lin and P. P. Vaidyanathan, "Application of DFT filter banks and cosine modulated filter banks in filtering," in Proc. APCCAS, Taipei, Taiwan, 1994, pp. 254-259.

[11] Z. Cvetković, "Oversampled modulated filter banks and tight Gabor frames in $l^{2}(\mathbb{Z}), "$ in Proc. IEEE ICASSP, Detroit, MI, May 1995, pp. $1456-1459$

[12] Z. Cvetković and M. Vetterli, "Oversampled filter banks," IEEE Trans. Signal Processing, vol. 46, pp. 1245-1255, May 1998.

[13] R. N. J. Veldhuis, "A filter-notation for analysis/synthesis systems and its relation to frames," Inst. Perception Res., Eindhoven, The Netherlands, Tech. Rep. 909, Sept. 1993.

[14] H. Bölcskei and F. Hlawatsch, "Frame-theoretic analysis and design of filter banks-Part I: Theory of uniform filter bank frames," Dept. Commun. Radio Freq. Eng., Vienna Univ. Technol., Vienna, Austria, Tech. Rep. 95-02/I, Nov. 1995.

[15] _ " "Frame-theoretic analysis and design of filter banks-Part II: Oversampled and critically sampled uniform filter banks," Dept Commun. Radio Freq. Eng., Vienna Univ. Technol., Vienna, Austria, Tech. Rep. 95-02/II, Nov. 1995.

[16] H. Bölcskei, F. Hlawatsch, and H. G. Feichtinger, "Oversampled FIR and IIR DFT filter banks and Weyl-Heisenberg frames," in Proc. IEEE ICASSP, Atlanta, GA, May 1996, vol. 3, pp. 1391-1394.

[17] _ _ "Frame-theoretic analysis and design of oversampled filter banks," in Proc. IEEE ISCAS, Atlanta, GA, May 1996, vol. 2, pp. 409-412.

[18] M. Vetterli and Z. Cvetković, "Oversampled FIR filter banks and frames in $l^{2}(\mathbb{Z})$," in Proc. IEEE ICASSP, May 1996, vol. 3, pp. 1530-1533.

[19] A. J. E. M. Janssen, "Density theorems for filter banks," Philips Res. Labs., Eindhoven, The Netherlands, Tech. Rep. 6858, Apr. 1995.

[20] Z. Cvetković, "Overcomplete expansions for digital signal processing," Ph.D. dissertation, Univ. California, Berkeley, Dec. 1995.

[21] H. Bölcskei, "Oversampled filter banks and predictive subband coders," Ph.D. dissertation, Vienna Univ. Technol., Vienna, Austria, Nov. 1997.

[22] H. Bölcskei and F. Hlawatsch, "Oversampled filter banks: Optimal noise shaping, design freedom, and noise analysis," in Proc. IEEE ICASSP, Munich, Germany, Apr. 1997, vol. 3, pp. 2453-2456.

[23] _ "Noise reduction in oversampled filter banks using predictive quantization," submitted for publication

[24] _ "Oversampled modulated filter banks," in Gabor Analysis and Algorithms: Theory and Applications, H. G. Feichtinger and T. Strohmer, Eds. Boston, MA: Birkhäuser, 1998, ch. 9, pp. 295-322.

[25] H. G. Feichtinger and T. Strohmer, Eds., Gabor Analysis and Algorithms: Theory and Applications. Boston, MA: Birkhäuser, 1998.

[26] H. Bölcskei and F. Hlawatsch, "Oversampled cosine modulated filter banks with perfect reconstruction," IEEE Trans. Circuits Syst. II, Special Issue on Multirate Systems, Filter Banks, Wavelets, and Applications, vol. 45, pp. 1057-1071, Aug. 1998.

[27] R. J. Duffin and A. C. Schaeffer, "A class of nonharmonic Fourier series," Trans. Amer. Math. Soc., vol. 72, pp. 341-366, 1952.

[28] I. Daubechies, Ten Lectures on Wavelets. Philadelphia, PA: SIAM, 1992.

[29] R. M. Young, An Introduction to Nonharmonic Fourier Series. New York: Academic, 1980.

[30] C. E. Heil and D. F. Walnut, "Continuous and discrete wavelet transforms," SIAM Rev., vol. 31, pp. 628-666, Dec. 1989.

[31] C. E. Heil, "Wavelets and frames," in Signal Processing-Part I: Signal Processing Theory, The IMA Volumes in Mathematics and its Applications, L. Auslander, Ed. New York: Springer, 1990, pp. 147-160.

[32] I. Daubechies, "Painless nonorthogonal expansions," J. Math. Phys., vol. 27, pp. 1271-1283, May 1986.

[33] _ "The wavelet transform, time-frequency localization and signal analysis," IEEE Trans. Inform. Theory, vol. 36, pp. 961-1005, Sept. 1990.

[34] M. Zibulski and Y. Y. Zeevi, "Oversampling in the Gabor scheme," IEEE Trans. Signal Processing, vol. 41, pp. 2679-2687, Aug. 1993.

[35] _ "Analysis of multiwindow Gabor-type schemes by frame methods," Appl. Comput. Harmon. Anal., vol. 4, pp. 188-221, Apr. 1997.

[36] _ "Multi-window Gabor-type transform for signal representation and analysis," in SPIE Proc. Wavelet Applications Signal Image Processing III, San Diego, CA, July 1995, pp. 116-127.

[37] N. J. Munch, "Noise reduction in tight Weyl-Heisenberg frames," IEEE Trans. Inform. Theory, vol. 38, pp. 608-616, Mar. 1992.

[38] P. P. Vaidyanathan, "Theory and design of $M$-channel maximally decimated quadrature mirror filters with arbitrary $M$, having perfect reconstruction property," IEEE Trans. Acoust., Speech, Signal Processing, vol. ASSP-35, pp. 476-492, Apr. 1987.

[39] H. Bölcskei and F. Hlawatsch, "Discrete Zak transforms, polyphase transforms, and applications," IEEE Trans. Signal Processing, vol. 45, pp. 851-866, Apr. 1997.
[40] A. J. E. M. Janssen, "The Zak transform: A signal transform for sampled time-continuous signals," Philips J. Res., vol. 43, no. 1, pp. 23-69, 1988.

[41] C. Heil, "A discrete Zak transform," Tech. Rep. MTR-89W000128, The MITRE Corp., Bedford, MA, Dec. 1989.

[42] L. Auslander, I. C. Gertner, and R. Tolimieri, "The discrete Zak transform application to time-frequency analysis and synthesis of nonstationary signals," IEEE Trans. Signal Processing, vol. 39, pp. 825-835, Apr. 1991.

[43] M. Vetterli and C. Herley, "Wavelets and filter banks: Theory and design," IEEE Trans. Signal Processing, vol. 40, pp. 2207-2232, Sept. 1992.

[44] A. W. Naylor and G. R. Sell, Linear Operator Theory in Engineering and Science, 2nd ed. New York: Springer, 1982.

[45] A. Ron and Z. Shen, "Frames and stable bases for shift-invariant subspaces of $L_{2}\left(\mathbb{R}^{d}\right)$," Can. J. Math., vol. 47, no. 5, pp. 1051-1094, 1995.

[46] D. G. Luenberger, Optimization by Vector Space Methods. New York: Wiley, 1969.

[47] A. Ben-Israel and T. N. E. Greville, Generalized Inverses: Theory and Applications. New York: Wiley, 1974.

[48] H. G. Feichtinger, "Pseudo-inverse matrix methods for signal reconstruction from partial data," in Proc. SPIE Conf. Visual Commun. Image Process., Boston, MA, Nov. 1991, pp. 766-772.

[49] O. Christensen, "Frame decompositions in Hilbert spaces," Ph.D. dissertation, Aarhus Univ., Aarhus, Denmark; Univ. Vienna, Vienna, Austria, 1993.

[50] T. A. C. M. Claasen, W. F. G. Mecklenbräuker, J. B. H. Peek, and N. V. Hurck, "Signal processing method for improving the dynamic range of A/D and D/A converters," IEEE Trans. Acoust., Speech, Signal Processing, vol. ASSP-28, pp. 529-538, 1980.

[51] V. K. Goyal, M. Vetterli, and N. T. Thao, "Quantized overcomplete expansions in $\mathbb{R}^{N}$ : Analysis, synthesis and algorithms," IEEE Trans. Inform. Theory, vol. 44, pp. 16-31, Jan. 1998.

[52] Z. Cvetković and M. Vetterli, "Overcomplete expansions and robustness," in Proc. IEEE TFTS, Paris, France, June 1996, pp. 325-328.

[53] K. Gröchenig, "Acceleration of the frame algorithm," IEEE Trans. Signal Processing, vol. 41, pp. 3331-3340, Dec. 1993.

[54] H. Bölcskei, F. Hlawatsch, and H. G. Feichtinger, "Equivalence of DFT filter banks and Gabor expansions," in Proc. SPIE Wavelet Appl. Signal Image Process. III, San Diego, CA, July 1995, vol. 2569, pt. I, pp. 128-139.

[55] V. Belevitch, Classical Network Theory. San Francisco, CA: HoldenDay, 1968.

[56] D. C. Youla, "On the factorization of rational matrices," IRE Trans. Inform. Theory, vol. IT-7, pp. 172-189, 1961

[57] I. Daubechies, S. Jaffard, and J. L. Journé, "A simple Wilson orthonormal basis with exponential decay," SIAM J. Math. Anal., vol. 22, pp. 554-572, 1991

[58] P. Vary, "On the design of digital filter banks based on a modified principle of polyphase," AË̈, vol. 33, nos. 7/8, pp. 293-300, 1979.

[59] A. J. E. M. Janssen, "Duality and biorthogonality for Weyl-Heisenberg frames," J. Fourier Anal. Appl., vol. 1, no. 4, pp. 403-436, 1995.

[60] H. Bölcskei, H. G. Feichtinger, and F. Hlawatsch, "Diagonalizing the Gabor frame operator," in Proc. IEEE UK Symp. Appl. Time-Freq. Time-Scale Methods, Univ. Warwick, Coventry, U.K., Aug. 1995, pp. 249-255a.

[61] F. Hlawatsch and H. Bölcskei, "Time-frequency analysis of frames," in Proc. IEEE-SP Int. Symp. Time-Freq. Time-Scale Anal., Philadelphia, PA, Oct. 1994, pp. 52-55.

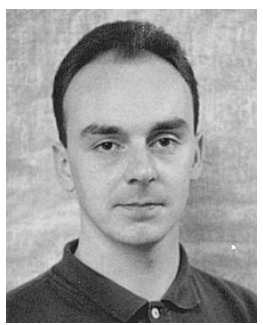

Helmut Bölcskei (S'94-M'98) was born in Austria on May 29, 1970. He received the Dipl.-Ing and Dr. Techn. degrees from Vienna University of Technology, Vienna, Austria, in 1994 and 1997, respectively.

From October to November 1994, he was a Researcher with the Department of Mathematics, University of Vienna. Since December 1994, he has been with the Department of Communications and Radio-Frequency Engineering, Vienna University of Technology, where he currently holds a Research and Teaching Assistant position. From February to May 1996, he was a Visiting Researcher with the Applied Mathematics Group, Philips Research Laboratories Eindhoven, The Netherlands, where he worked on video coding. From February to March 1998, he visited ENST Paris, Paris, France, where he was involved in research on multicarrier modulation. His research interests include signal processing for communications and information theory. 


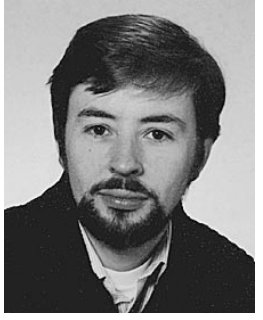

Franz Hlawatsch (S'85-M'88) received the Dipl.Ing., Dr. Techn., and Univ.-Dozent degrees in electrical engineering/signal processing from the Vienna University of Technology, Vienna, Austria, in 1983, 1988, and 1996, respectively.

Since 1983, he has been with the Department of Communications and Radio-Frequency Engineering, Vienna University of Technology. From 1991 to 1992, he spent a sabbatical year with the Department of Electrical Engineering, University of Rhode Island, Kingston. He authored the book Time-Frequency Analysis and Synthesis of Linear Signal Spaces-TimeFrequency Filters, Signal Detection and Estimation, and Range-Doppler Estimation (Boston, MA: Kluwer, 1998) and coedited the book The Wigner Distribution-Theory and Applications in Signal Processing (Amsterdam, The Netherlands: Elsevier, 1997). His research interests are in signal processing with emphasis on time-frequency methods.

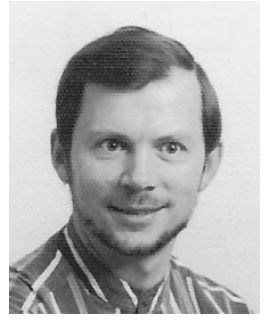

Hans G. Feichtinger was born June 16, 1951, in Wr. Neustadt, Austria. He studied mathematics and physics at the University of Vienna, Vienna, Austria from 1969 to 1974 and received the Ph.D. degree in mathematics in 1974.

He held habilitation in 1979 and visiting positions in Nancy, France, in 1980, in Heidelberg, Germany, in 1980, in College Park, MD, for the academic year 1989 to 1990 , at the Mittag-Leffler Institute, Stockholm, Sweden, in April 1992, in Marseille, France, in June 1992, and in Storrs, CT, for the spring term of 1994. He has participated in approximately 120 talks at mathematical and applied conferences or invited lectures and has authored more than 100 works in pure and applied mathematics. Together with $\mathrm{T}$. Strohmer, he is the co-editor of Gabor Analysis and Algorithms-Theory and Applications (Boston, MA: Birkhäuser, 1998). He is Group Leader of Numerical Harmonic Analysis Group, Mathematics Department, University of Vienna. His main fields of interest are irregular sampling, scattered data approximation, Gabor expansions, wavelet theory, nonorthogonal expansions, time-frequency localization, and signals of variable bandwidth

Dr. Feichtinger is a member of EMS, AMS, SIAM, GAMM, SPIE, OEMG, OEAGM, and DMV. 